\title{
MHSalud
}

ISSN: 1659-097X

revistamhsalud@una.cr

Universidad Nacional

Costa Rica

\section{Gasto energético e intensidad del entrenamiento de porrismo: Un estudio descriptivo}

Ulloa Sánchez, Paul; Hernández Elizondo, Jessenia

Gasto energético e intensidad del entrenamiento de porrismo: Un estudio descriptivo

MHSalud, vol. 18, núm. 2, 2021

Universidad Nacional, Costa Rica

Disponible en: https://www.redalyc.org/articulo.oa?id=237066090008

DOI: https://doi.org/10.15359/mhs.18-2.8

\section{(c) (1) () 9}

Esta obra está bajo una Licencia Creative Commons Atribución-NoComercial-SinDerivar 3.0 Internacional. 


\section{Gasto energético e intensidad del entrenamiento de porrismo: Un estudio descriptivo}

\section{Energy Expenditure and Intensity of Cheerleading Training: A Descriptive Study}

Gasto de energia e intensidade do treinamento da equipe de torcida: Um estudo descritivo

Paul Ulloa Sánchez

Universidad de Costa Rica, Costa Rica

piuullo@gmail.com

(iD) https://orcid.org/0000-0002-1374-7192

Jessenia Hernández Elizondo

Universidad de Costa Rica, Costa Rica

jessenia.hernandez@ucr.ac.cr

https://orcid.org/0000-0001-9295-129X
DOI: https://doi.org/10.15359/mhs.18-2.8

Redalyc: https://www.redalyc.org/articulo.oa?

$\mathrm{id}=237066090008$

Recepción: 24 Junio 2020

Aprobación: 11 Febrero 2021

\section{RESUMEN:}

El objetivo del presente estudio fue aclarar las siguientes preguntas: ¿̇on equivalentes los gastos energéticos en términos de METs de los entrenamientos de porrismo y lo reportado por el compendio de gasto energético de Ainsworth et al. (2011)? ¿ Hay diferencias estadísticamente significativas del gasto energético e intensidad del entrenamiento de porrismo al comparar diferentes categorías de rendimiento y sexo?

Metodología: Se midió el gasto energético y la intensidad durante una hora de entrenamientos de porrismo, a una muestra compuesta por 103 porristas (hombres $=55$; mujeres $=48$ ), los cuales pertenecen a tres equipos all stars y dos selecciones nacionales de Costa Rica.

Resultados: No se encontraron diferencias entre el valor reportado por Ainsworth et al. (2011) y los entrenamientos de porrismo (6 METs vs 5.8 METs, $\mathrm{t}=-1.7, \mathrm{p}=0.092)$. Se evidenció una interacción significativa del gasto energético $(\mathrm{kcal})$ entre sexo y categoría de rendimiento.

Conclusiones: El gasto energético en términos de METs de los entrenamientos de porrismo es equivalente a lo reportado por Ainsworth et al. (2011). Los hombres con categoría "alta" de rendimiento tuvieron un gasto energético significativamente mayor $\left(\mathrm{p}<0.05^{*}\right)$ comparado con los hombres de categoría "media" o "baja" y con las mujeres de las tres categorías. No se encontraron diferencias en términos de intensidad al comparar sexo y categoría de rendimiento.

Palabras Clave: Ejercicio físico, atletas, rendimiento.

\section{ABstract:}

The objective of the present study was to clarify the following questions: are equivalent the energy expenditures in terms of METs of cheerleading training sessions and that shown by Ainsworth et al. (2011)? Are there statistically significant differences in the energy expenditure and intensity of cheerleading training when comparing different performance and sex categories?

Methodology: Energy expenditure and intensity were measured during one hour of cheerleading training, for a sample of 103 cheerleaders $($ men $=55$; women $=48)$, who belong to three all-star teams and two national teams from Costa Rica.

Results: No differences were found between the value reported by Ainsworth et al. (2011) and cheerleading workouts (6 METs vs. $5.8 \mathrm{MET}, \mathrm{t}=-1.7, \mathrm{p}=0.092$ ). A significant interaction of energy expenditure (kcal) between sex and performance category was evident.

Conclusions: The energy expenditure in terms of METs for cheerleading workouts is equivalent to that reported by Ainsworth et al. (2011). Men with a "high" performance category had a significantly higher energy expenditure $\left(\mathrm{p}<0.05^{*}\right)$ compared to men with a "medium" or "low" category and with women in all three categories. No differences were found in terms of intensity when comparing sex and performance category.

KEYWORDS: physical exercise, athletes, performance.

\section{Resumo:}

O objetivo do presente estudo foi para esclarecer as seguintes questões: os gastos de energia são equivalentes em termos de METs de treinamento de equipe de torcida e os relatados pelo compêndio de gasto de energia de Ainsworth et al. (2011). Existem diferenças 
estatisticamente significativas no gasto de energia e na intensidade do treinamento da equipe ao comparar diferentes categorias de desempenho e gênero?

Metodologia: o gasto energético e a intensidade foram medidos durante uma hora de treinamento da equipe de torcida, em uma amostra composta por 103 líderes de torcida (homens $=55$; mulheres $=48$ ), pertencentes a três equipes. todas as estrelas e duas seleções da Costa Rica.

Resultados: não foram encontradas diferenças entre o valor relatado por Ainsworth et al. (2011) e treinos de equipes de torcida (6 METs vs 5,8 METs, $\mathrm{t}=-1,7, \mathrm{p}=0,092$ ). Evidenciou-se interação significativa do gasto energético (kcal) entre sexo e categoria de desempenho.

Conclusão: $\mathrm{O}$ gasto energético em termos de METs do treinamento da equipe de torcida é equivalente ao relatado por Ainsworth et al. (2011). Os homens na categoria "alto" de desempenho tiveram um gasto energético significativamente maior $\left(\mathrm{p}<0,05^{*}\right)$ em comparação com os homens na categoria "médio" ou "baixo" e com as mulheres nas três categorias. Não foram encontradas diferenças em termos de intensidade ao comparar sexo e categoria de desempenho.

Palavras-Chave: exercício físico, atletas, desempenho.

\section{INTRODUCCIÓN}

El porrismo (cheerleading) es un deporte que tiene sus orígenes a finales del siglo XIX en las universidades de los Estados Unidos. Su historia es extensa e interesante, pero lo que más llama la atención es su expansión por todo el mundo, a tal punto de formar una organización mundial (International Cheer Union - ICU) con 116 federaciones nacionales afiliadas en el 2020 (International Cheer Union [ICU], 2020).

Las competencias de porrismo consisten en la realización de una rutina de 2 min y $30 \mathrm{~s}$, esta se compone de diferentes partes, donde las porristas y los porristas tienen funciones específicas de acuerdo a sus habilidades y composición corporal. Las posiciones más importantes son la base y la flyer: la base es el atleta que sostiene o lanza a la flyer y, por su parte, la flyer es la que sube y ejecuta los elementos en la parte más alta de los elementos de construcción. Los elementos de construcción son principalmente las parejas o stunts (estructuras constituidas por una sola flyer y una o varias bases); las pirámides, que son estructuras compuestas por todos los integrantes del equipo y donde las flyers llegan a colocarse por encima de hasta dos o tres compañeros o compañeras acomodados verticalmente, y las basket toss consisten en el lanzamiento de las flyers por parte de las bases lo más alto posible. En las rutinas también se incluye gimnasia (gimnasia de piso, como: rodada, flic-flac, mortal, entre otros); la totalidad de integrantes del equipo debe realizarla.

Las rutinas de porrismo exigen un alto nivel de fuerza explosiva de extremidades superiores e inferiores, además de estabilidad corporal para una ejecución perfecta de sus elementos, por lo que las personas atletas de este deporte suelen ser físicamente muy completas, principalmente cuando llevan un proceso formativo desde las categorías básicas hasta las más altas.

En este deporte, las competencias se organizan por nivel y categorías. En la actualidad existen los niveles del 1 al 7, el 1 es el más básico y el 7 el de mayor dificultad técnica. El nivel de dificultad de una rutina lo define principalmente la complejidad de los elementos de construcción y de la gimnasia de piso, es decir, en cada nivel se pueden ejecutar los elementos que el reglamento le permite; cada equipo define su nivel de competencia dependiendo de las capacidades técnicas de sus atletas. Las categorías que se incluyeron en el presente estudio fueron clubes (también conocidos como all stars) y selecciones nacionales; también existen los equipos institucionales: su principal característica es que lo conforman estudiantes de un centro educativo específico y tienen una "porra" que les identifica. Comúnmente estos atletas reciben becas de estudio por representar a la institución; incluso, algunas instituciones son muy reconocidas principalmente por su equipo de porrismo.

Ahora bien, a pesar de que el porrismo es un deporte que se ha expandido por muchos países, en la actualidad no se conoce de algún estudio científico que muestre el gasto energético e intensidad que se experimenta al practicarlo, como sí se ha hecho con otros deportes y/o pruebas físicas, por ejemplo: se ha visto que ejercicios globales de baloncesto alcanzan una intensidad del $87 \%$ al $91 \%$ de la frecuencia cardiaca 
máxima (Delextrat y Kraiem, 2013); además, se ha visto que el baloncesto es de una intensidad vigorosa y que los principales sistemas energéticos en dicho deporte son los de la vía anaeróbica (Latzel et al., 2018); por otro lado, también se sabe que el entrenamiento en trampolín es una actividad física vigorosa, pues se tienen reportes de que el equivalente metabólico de una muestra específica llegó hasta los 5.2 METs y, se registró un gasto energético promedio de $317 \mathrm{kcal}$ en una sesión de entrenamiento de $50 \mathrm{~min}$ (Cugusi et al., 2017). En este sentido, el compendio desarrollado por Ainsworth et al. (2011) reporta que la práctica de porrismo y los movimientos gimnásticos elevan el equivalente metabólico a $6 \mathrm{METs}$, que es actividad física vigorosa (US Department of Health and Human Services, 2008).

Como se mencionó, no se tiene conocimiento de algún estudio científico enfocado en la medición del gasto energético e intensidad de la práctica del porrismo, por lo que para el presente estudio se plantearon dos preguntas de investigación: ¿Son equivalentes los gastos energéticos en términos de METs los entrenamientos de porrismo y lo reportado por el compendio de gasto energético de Ainsworth et al. (2011)? ¿Hay diferencias estadísticamente significativas del gasto energético e intensidad del entrenamiento de porrismo al comparar diferentes categorías de rendimiento y sexo?

\section{Metodología}

\section{Participantes}

Participaron en el presente estudio porristas activos $(n=103$; hombres $=55$; mujeres $=48)$ de tres equipos all stars y dos selecciones nacionales de Costa Rica. La tabla 1 muestra las características que describen a la población participante en el presente estudio. El rango de edad de los equipos all stars es de 15 a 36 años, el de la selección nacional Coed de 14.8 a 34 años y, el de la selección nacional Junior 11.8 a 16.7 años. El rango de edad de toda la muestra es de 11.8 a 36 años.

TABLA 1

Datos descriptivos de la muestra utilizada en el presente estudio

\begin{tabular}{clccccc}
\hline Sexo & & AS1 & AS2 & AS3 & S1 & S2 \\
\hline \multirow{6}{*}{ Hombres } & $\mathrm{N}$ & 13 & 11 & 12 & 4 & 15 \\
& Edad (años) & $24.1 \pm 3.2$ & $23.5 \pm 6.1$ & $25 \pm 4.4$ & $16.1 \pm 0.7$ & $26.9 \pm 4.2$ \\
& Talla (cm) & $173.9 \pm 8.5$ & $171.5 \pm 3.2$ & $174.3 \pm 7.1$ & $172.3 \pm 10.2$ & $174.7 \pm 5.9$ \\
& Masa corporal (kg) & $77.79 \pm 16.1$ & $74.1 \pm 12.3$ & $79.7 \pm 15.9$ & $75.15 \pm 8.4$ & $85.8 \pm 19.9$ \\
& & & & & & \\
\multirow{6}{*}{ Mujeres } & $\mathrm{N}$ & 7 & 8 & 11 & 13 & 9 \\
& Edad (años) & $20.3 \pm 2.8$ & $18.5 \pm 2.1$ & $23 \pm 3.3$ & $13.9 \pm 1.4$ & $19.5 \pm 4.28$ \\
& Talla (cm) & $159.4 \pm 5.1$ & $154.6 \pm 2.8$ & $155.7 \pm 6$ & $154.5 \pm 7.3$ & $152.9 \pm 3$ \\
& Masa corporal (kg) & $52.3 \pm 4.3$ & $48.4 \pm 4.44$ & $51.4 \pm 8.5$ & $48.8 \pm 11.1$ & $49.3 \pm 6.2$ \\
\hline
\end{tabular}

Nota: AS1 = equipo all stars 1, AS2 = equipo all stars 2, AS3 = equipo all stars 3, $S 1$ = selección nacional 1 y $S 2=$ selección nacional 2 . Fuente: Elaboración propia.

\section{Criterios de inclusión}

Con el objetivo de homogenizar la muestra, se incluyeron equipos de porrismo conformados por deportistas capaces de ejecutar, como mínimo, las siguientes destrezas específicas:

1. Gimnasia de piso estática: flic-flac o mortal.

2. Gimnasia de piso corrida: rodada, flic-flac, mortal. 
3. Rutina que incluya el elemento full en la parte de construcción.

\section{Categorización de rendimiento de cada atleta}

La categorización de rendimiento se realizó tomando en cuenta principalmente su nivel de gimnasia, debido a que, en porrismo, el nivel de gimnasia de piso es una variable esencial para determinar la exigencia física que un atleta específico cumple dentro de una rutina. Si bien es cierto, lo ideal es que todos y todas en un equipo dominen la máxima dificultad de gimnasia para su categoría y nivel en el que compite, esto es muy difícil de cumplir por diferentes razones (años de preparación, capacidad de aprendizaje de las acrobacias, entre otras). Los elementos gimnásticos que se tomaron en cuenta son los que se muestran a continuación en la tabla 2.

TABLA 2

Categorización del nivel de gimnasia

\begin{tabular}{ccc}
\hline Nivel & Gimnasia estática & Gimnasia corrida \\
\hline 1 & Ejecuta mortal o no ejecuta mortal & No ejecuta nada \\
2 & Ejecuta mortal & Ejecuta rodada flic o rondada flic mortal \\
3 & Ejecuta mortal o ejecuta mortal con giro & Ejecuta rondada flic mortal con giro \\
\hline
\end{tabular}

Fuente: Elaboración propia.

\section{Entrenamiento de rutinas completas}

En el contexto del porrismo existen los llamados entrenamientos de rutinas completas; estos se desarrollan en la etapa final del macrociclo de entrenamiento y se caracterizan principalmente por la ejecución de la rutina repetidamente durante todo el entrenamiento. En las primeras repeticiones se ejecutan pocos elementos haciendo énfasis en los tiempos, a lo que también se llama "marcar la rutina", pero conforme van avanzando las repeticiones se ejecutan más partes de la rutina hasta llegar a ejecutar una, dos o hasta tres "full outs" o rutinas con la ejecución de todas sus partes. Estos entrenamientos generalmente duran 2 horas y suelen ser los más exhaustivos comparados con los del inicio del macrociclo. En el presente estudio se estableció una constante para la recolección de datos, la cual fue utilizar los datos de la última hora de los entrenamientos de rutinas completas de los equipos reclutados.

\section{Lugares de recolección de datos}

Los datos se recolectaron en las instalaciones en las que los equipos de porrismo entrenan sus rutinas completas. Son gimnasios amplios con espacio para el mat o piso especial para porrismo, el cual está conformado por nueve franjas unidas, que forman un rectángulo de $18 \mathrm{~m}$ por $12 \mathrm{~m}$ aproximadamente (figura $1)$. 


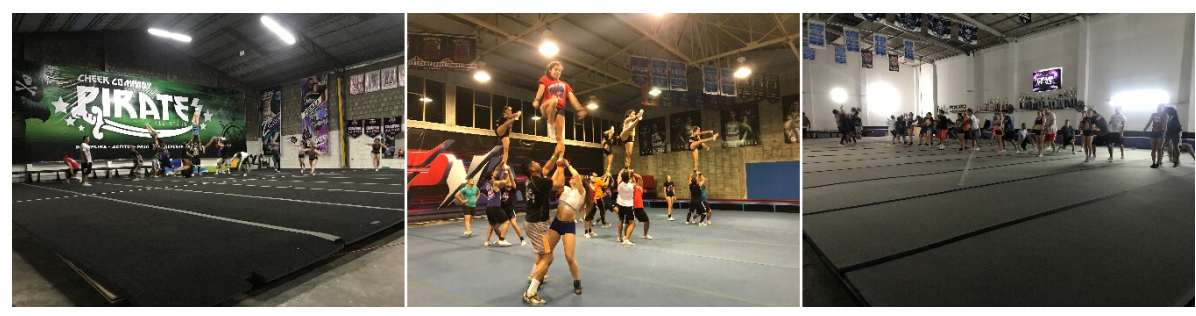

FIGURA 1

Representación de los centros de entrenamiento de porrismo.

Nota: Elaboración propia.

\section{Instrumentos de medición}

Para medir la composición corporal se utilizó un analizador de composición corporal por bioimpedancia marca InBody modelo DSM-BIA Multifrecuencia Segmental (InBody, Seúl, Corea del Sur).

Para recolectar la información de los entrenamientos de rutinas completas se emplearon acelerómetros triaxiales (medición en 3 ejes) ActiGraph modelos wGT3X-BT (ActiGraph, Maastricht, Bélgica). Estos dispositivos muestran los METs y, a partir de esta unidad de medición, también calculan el gasto calórico (kcal). Los METs son la unidad de medida del equivalente metabólico; corresponde a $3.5 \mathrm{ml} / \mathrm{kg}$ min, el cual es el consumo mínimo de oxígeno que el organismo necesita para mantener sus funciones vitales. Un rango de 1.0-1.5 METs se evalúa como conducta sedentaria, de 1.6-2.9 METs como actividad de intensidad baja, de 3-5.9 como actividad de intensidad moderada, y $>6$ METs como actividad de intensidad vigorosa.

Estudios como el de Cugusi et al. (2017) también han utilizado acelerómetros para medir un entrenamiento determinado, con una duración aproximada de $46.1 \pm 5.0 \mathrm{~min}$; esta duración es similar a la establecida en el presente estudio.

La colocación de los acelerómetros fue establecida por los investigadores tomando en cuenta las características del deporte, las mujeres (flyers) se lo colocaron en la muñeca, debido a que la zona de la cintura tiene más roce con los compañeros, mientras que los hombres (bases) se lo colocaron en la cintura, ya que, contrario a las flyers, la zona de las muñecas tiene mayor contacto con los compañeros durante la práctica del porrismo. En relación a este aspecto, Leppänen et al. (2019) demostraron que la colocación de los acelerómetros en la muñeca y en la cintura muestran asociaciones consistentes, es decir, los datos recolectados no difieren, o no se ven afectados si se colocan en alguna de estas ubicaciones. Es importante destacar que los acelerómetros que utilizaron Leppänen et al. (2019) son los mismos que se utilizaron en el presente estudio.

Además, se utilizaron pulsómetros Polar modelo RS200sd (Polar Electro, Kempele, Finlandia) para medir la frecuencia cardiaca promedio (FCP), frecuencia cardiaca máxima (FCmax) y el gasto calórico (kcal). Debido a la disponibilidad de los pulsómetros, no todos los sujetos pudieron ser monitoreados, por lo que aleatoriamente se seleccionó a la mitad de los atletas, en cada recolección de datos.

Estudios como el de Cugusi et al. (2017), Delextrat y Kraiem, (2013), Latzel et al. (2018) y Zunzer et al. (2013) también han utilizado pulsómetros para medir diferentes pruebas deportivas, como un entrenamiento en trampolín, entrenamiento de baloncesto y rondas de golf.

\section{Procedimiento}

El reclutamiento de los sujetos se realizó bajo la autorización de los entrenadores y administrativos de los centros deportivos Pura Vida Athletics, Jungle Cats, Cheer Company Pirates y de la Federación Cheer and Dance Costa Rica (FECAD). 
Antes de iniciar la recolección de datos, el investigador y la investigadora realizaron una prueba piloto para establecer la ubicación de los instrumentos de medición; a partir de dichas pruebas se determinó que, para evitar interrumpir demasiado el entrenamiento normal, se debían colocar los acelerómetros antes de empezar el entrenamiento y, una hora antes de que terminara el entrenamiento se les colocaron los pulsómetros. Los acelerómetros se activan a la hora que se les programó en el momento de su codificación. Cada recolección de datos se desarrolló de la siguiente forma: como ya se mencionó, cada atleta se colocó el acelerómetro donde le corresponde (a las bases en la muñeca y a las flyers en la cintura), luego el grupo de atletas inició con su entrenamiento normal (calentamiento, repaso técnico y coreográfico, etc.), una hora antes de la finalización del entrenamiento se les colocaron los pulsómetros y, al finalizar el entrenamiento, los atletas entregaron los instrumentos de medición. Por último, de parte del equipo investigador se les agradeció la participación en el estudio.

\section{Variables}

En la tabla 3 se muestran las variables establecidas para el presente estudio junto con su respectiva definición operacional.

TABLA 3

Variables establecidas para la realización del estudio

\begin{tabular}{ccc}
\hline & Variables & Definición operacional \\
\hline Independientes & Categoría & Rendimiento deportivo (categoría de gimnasia 1, 2 y 3) \\
& Sexo & Género masculino y femenino \\
& Equivalente metabólico (METs) & Promedio de METs registrados en una hora de EP \\
Dependientes & Gasto calórico (kcal) & Kcal gastadas en una hora de EP \\
& Intensidad en FCP & Latidos por minuto promedio registrados en una hora de EP \\
& Intensidad en FCmax & Latidos por minuto máximo alcanzado en una hora de EP \\
\hline
\end{tabular}

Nota: $\mathrm{kcal}=$ kilocalorías, FCP $=$ frecuencia cardiaca promedio, $\mathrm{FCmax}=$ frecuencia cardiaca máxima, EP = entrenamiento de porrismo. Fuente: Elaboración propia.

\section{Análisis estadístico}

Se calcularon promedio y desviación estándar para los datos descriptivos de los sujetos, METs, gasto calórico y FCmax.

A cada sujeto se le calculó la FCmax a partir de la fórmula propuesta por Tanaka et al. (2001): 208.75 (0.7 edad).

Se realizó una prueba t-Student para una muestra, para responder el primer problema de investigación. Además, se utilizó un análisis de varianza (ANOVA) de 2 vías para grupos independientes, para evidenciar diferencias entre categoría de rendimiento y sexo.

Adicionalmente, se realizó ANOVA de 1 vía para grupos independientes con ánimo de evidenciar diferencias significativas entre las mediciones de gasto energético con acelerómetro y pulsómetro. También se realizó correlación de Spearman para mostrar la relación entre ambos métodos de medición. 


\section{Resultados}

Los resultados de la comparación por medio de la prueba t-Student, para una muestra del gasto energético en términos de METs con el valor de referencia reportado por Ainsworth et al. (2011) (6 METs) en la práctica del porrismo, mostró que no existe diferencia significativa, utilizando los datos de la totalidad de la muestra del presente estudio $(\mathrm{N}=103, \operatorname{MET} s=5.8, \mathrm{t}=-1.7, \mathrm{p}=0.092)$.

Además, considerando la muestra según la edad de quienes practican porrismo, se evidenció que los menores de 18 años $(\mathrm{N}=29)$ tuvieron un gasto energético en términos de METs significativamente menor $\left(\right.$ METs $\left.=4.8, \mathrm{t}=-5.81, \mathrm{p} \leq 0.001^{*}\right)$ al del compendio de Ainsworth et al. (2011). Por otro lado, los mayores de 18 años $(\mathrm{N}=74)$ no mostraron diferencia significativa $($ METs $=5.5, \mathrm{t}=0.53, \mathrm{p}=0.593)$ con lo reportado en dicho compendio (Ver tabla 4 y 5 ).

\section{TABLA 4}

Comparación del gasto energético en términos de METs comparado con el valor de referencia reportado por Ainsworth (6 METs) en la práctica del porrismo, considerando la muestra según la edad de la persona deportista

\begin{tabular}{lcccc}
\hline \multicolumn{1}{c}{ Muestra } & N & METs $\bar{X}$ & t & p \\
\hline Todos y todas & 103 & 5.8 & -1.7 & 0.092 \\
Menores de 18 años & 29 & 4.8 & -5.81 & $\leq 0.001^{*}$ \\
Mayores de 18 años & 74 & 5.5 & -0.53 & 0.593 \\
\hline
\end{tabular}

\section{Nota: ${ }^{*}=$ diferencia estadísticamente significativa. Fuente: Elaboración propia.}

En cuanto a la variable de gasto energético en términos de kilocalorías (kcal), medidas con acelerómetros, la ANOVA de dos vías para grupos independientes mostró que existe interacción significativa entre sexo y categoría de gimnasia $\left(F=5.07, p=0.008^{*}\right)$; esta interacción se ilustra en la figura 2 . Por otro lado, esta misma variable, pero medida con pulsómetros, mostró una diferencia significativa entre sexos $(\mathrm{F}=4.08, \mathrm{p}$ $=0.048^{*}$ [figura 3]) pero no entre categoría de gimnasia $(\mathrm{F}=0.99, \mathrm{p}=0.377)$ y tampoco en interacción $(\mathrm{F}$ $=0.27, \mathrm{p}=0.764)$.

Por último, la variable de intensidad en términos de FCP no mostró diferencias significativas entre sexos $(\mathrm{F}=0.33, \mathrm{p}=0.568)$, categoría de gimnasia $(\mathrm{F}=0.82, \mathrm{p}=0.444)$ ni interacción significativa $(\mathrm{F}=0.28, \mathrm{p}=$ 0.760). En términos de FCmax tampoco hubo diferencias significativas entre sexos $(\mathrm{F}=0.38, \mathrm{p}=0.539) \mathrm{ni}$ categoría de gimnasia $(F=0.86, p=0.429)$; asimismo, no hubo interacción significativa $(F=0.30, p=0.738)$. 
MHSALUD, ISSN: 1659-097X, 18(2), JULIO-DiciembRe, 2021, PP 1-14

UlloA SÁNCHEZ, HeRNÁNDEZ Elizondo

TABLA 5

Interacción entre el sexo y el nivel de destreza de porristas, en variables de kilocalorías y FC

\begin{tabular}{|c|c|c|c|c|c|c|c|c|c|}
\hline \multirow{3}{*}{ Variables } & \multicolumn{3}{|c|}{ Hombres } & \multicolumn{3}{|c|}{ Mujeres } & \multicolumn{3}{|c|}{ ANOVA } \\
\hline & \multicolumn{3}{|c|}{ Nivel de gimnasia } & \multicolumn{3}{|c|}{ Nivel de gimnasia } & \multirow{2}{*}{$\begin{array}{l}\text { Sexo } \\
\text { (p) }\end{array}$} & \multirow{2}{*}{$\begin{array}{c}\text { Cat. de } \\
\text { gimnasia } \\
\text { (p) }\end{array}$} & \multirow{2}{*}{$\begin{array}{c}\text { Interacción } \\
\text { (p) }\end{array}$} \\
\hline & Bajo & Medio & Alto & Bajo & Medio & Alto & & & \\
\hline $\begin{array}{l}\text { Kcal } \\
\text { (Acelerómetro) }\end{array}$ & $180.4 \pm 43$ & $178.5 \pm 42$ & $300.9 \pm 132.7$ & $146.8 \pm 32$ & $153.5 \pm 43.3$ & $151.3 \pm 39.2$ & $\leq 0.001^{*}$ & $0.009 *$ & $0.008^{*}$ \\
\hline $\mathrm{N}$ & 9 & 16 & 30 & 5 & 35 & 8 & & & \\
\hline $\begin{array}{l}\text { Kcal } \\
\text { (pulsómetro) }\end{array}$ & $313.33 \pm 89$ & $382.3 \pm 158.3$ & $396.8 \pm 106.9$ & 203 & $189.9 \pm 14.5$ & $200 \pm 19.6$ & $0.048^{*}$ & 0.377 & 0.764 \\
\hline $\mathrm{FCP}$ & $121.7 \pm 7.8$ & $132.5 \pm 12$ & $133.5 \pm 10.2$ & 130 & $131.2 \pm 11.7$ & $134.9 \pm 12$ & 0.568 & 0.444 & 0.760 \\
\hline FCmax & $205 \pm 23.4$ & $200 \pm 21.5$ & $202.2 \pm 19$ & 203 & $189.9 \pm 14.5$ & $200 \pm 19.6$ & 0.539 & 0.429 & 0.738 \\
\hline $\mathrm{N}$ & 3 & 8 & 19 & 1 & 26 & 8 & & & \\
\hline
\end{tabular}

Nota: $\mathrm{kcal}=$ kilocalorías; FCP $=$ frecuencia cardiaca promedio,

FCmax $=$ frecuencia cardiaca máxima. Fuente: Elaboración propia.

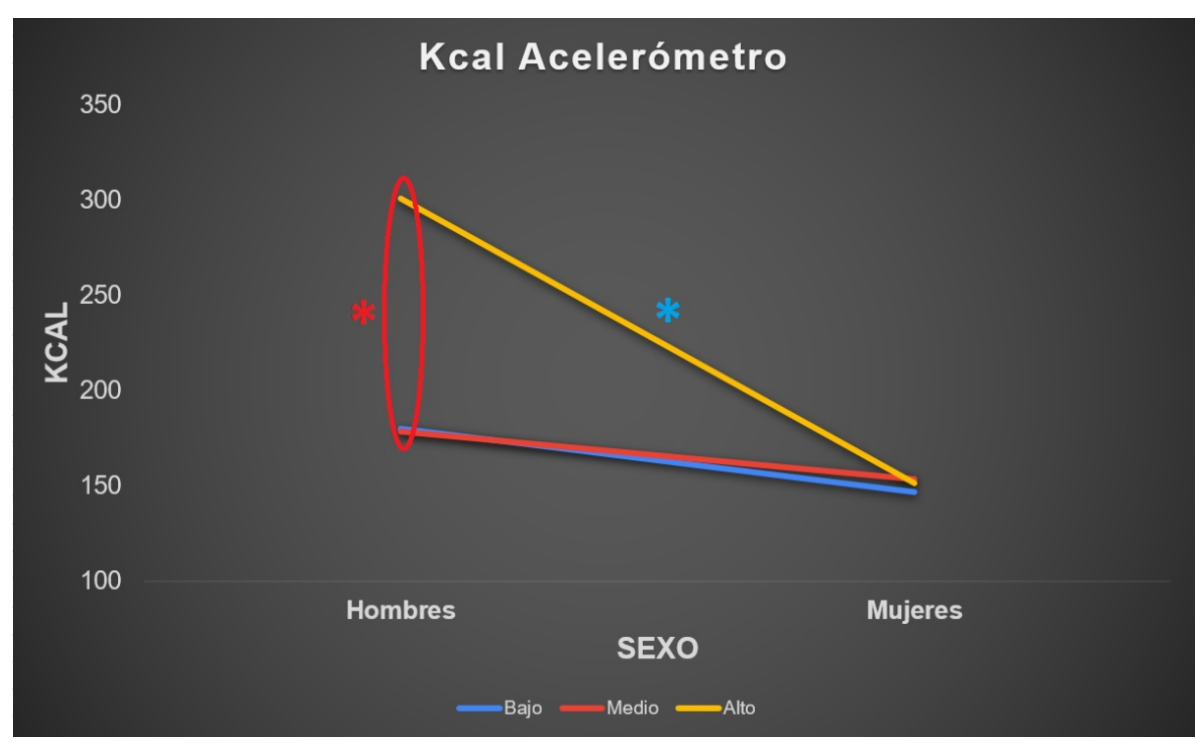

FIGURA 2

Gráfico de interacción doble entre categoría de gimnasia y sexo, de la variable gasto energético medido con el acelerómetro

Fuente: Elaboración propia. Nota: asterisco azul $=$ diferencia significativa $\left(\mathrm{p}<0.05^{*}\right)$ entre hombres y mujeres de categoría de gimnasia alta; asterisco rojo $=$ deferencia significativa $(\mathrm{p}$ $<0.05^{*}$ ) entre hombres de categoría de gimnasia alta y hombres de categoría media y baja. 


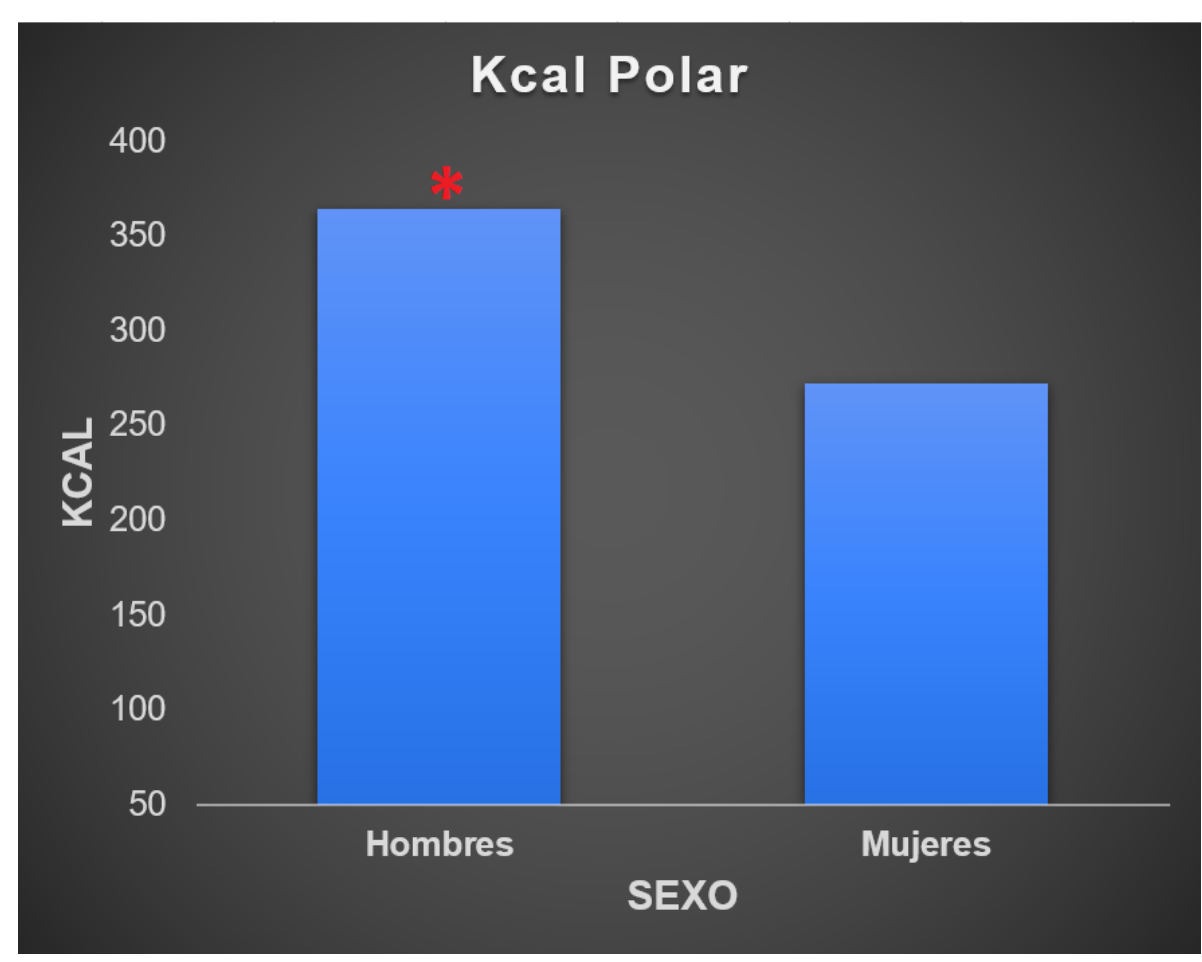

FIGURA 3

Gráfico de comparación entre sexo de las kcal registradas con los pulsómetros.

Fuente: Elaboración propia. Nota: asterisco $=$ diferencia significativa $\left(\mathrm{p}<0.05^{*}\right) ; \mathrm{N}$ hombres $=29, \mathrm{~N}$ mujeres $=35$.

\section{DiscuSIÓN}

Para responder la primera pregunta de investigación, la prueba t para una muestra evidenció que no existe diferencia significativa $(\mathrm{p}=0.092)$ entre el promedio de METs gastados durante los entrenamientos de porrismo registrados en el presente estudio $(\mathrm{N}=103$, METs $\overline{\mathrm{X}}=5.8)$ y el gasto energético reportado por Ainsworth et al. (2011), equivalente a 6 METs. Estos resultados demuestran que el entrenamiento de porrismo se clasifica como una actividad física vigorosa, que genera un gasto aproximado de $6 \mathrm{MET}$ s en una hora de práctica.

En este sentido, dicha equivalencia ayuda a establecer bases de evidencia científica más concretas acerca de la exigencia física que requiere el deporte del porrismo, ya que, hasta el momento, la evidencia de este tipo es poco abundante en las bases de datos científicas. Debe señalarse que algunos deportistas reportaron gastos energéticos por debajo del promedio, pero otros inclusive registraron un gasto metabólico superior a 7 y 8 $\operatorname{METs}(\mathrm{N}=7$ y $\mathrm{N}=2$ respectivamente).

Adicionalmente, es bueno conocer algunos gastos metabólicos en otros deportes, para tener una mejor referencia y crear una idea más clara de lo que es el entrenamiento de porrismo; por ejemplo, algunos deportes que también se clasifican como actividad física vigorosa son: baloncesto general (6.6 METs), tenis (6 METs) y rugby (6.3 METs) (Ainsworth et al., 2011).

Por otro lado, tomando en cuenta el amplio rango de edad de los sujetos incluidos en el presente estudio (11.8-36 años), y sabiendo que el compendio de Ainsworth et al. (2011) llevó a cabo sus estudios con sujetos mayores de 18 años, fue necesario realizar un análisis adicional, el cual evidenció que los METs de los sujetos menores de 18 años son significativamente más bajos $\left(\mathrm{N}=29\right.$, METs $\left.\overline{\mathrm{X}}=4.8, \mathrm{p} \leq 0.001^{*}\right)$ que lo reportado en el compendio, mientras que los mayores de 18 años no tuvieron un gasto metabólico durante el entrenamiento significativamente diferente $(\mathrm{N}=74$, METs $\bar{X}=5.5, \mathrm{p}=0.593)$ con respecto a los datos reportados en el compendio. Es decir, los resultados fueron equivalentes al del compendio. 
La diferencia significativa del gasto energético entre los menores de 18 años y el reportado en el compendio podría explicarse por dos factores, ambos relacionados a la masa corporal: en primera instancia se sabe que el gasto energético está estrechamente relacionado tanto con la masa corporal como con la masa libre de grasa; en el caso de las personas porristas jóvenes (menores de 18 años) generalmente an no han completado s u proceso de maduración; por ende, probablemente tengan mucho menos masa muscular en comparación con los mayores de 18 años (Lyden et al., 2013; Zakeri et al., 2006). En segunda instancia las diferencias podrían justificarse por un factor mecánico, es decir, entre mayor masa corporal tenga el sujeto mayor energía r equiere para desplazarse y ejecutar los complejos movimientos y fundamentos técnicos del porrismo. Por consiguiente, los sujetos menores de 18 años (masa corporal $\bar{X}=55.04 \mathrm{~kg}, \mathrm{~N}=29$ ), al tener menor peso corporal que trasladar con respecto a los mayores de 18 años (masa corporal $\bar{X}=69.63 \mathrm{~kg}, \mathrm{~N}=$ 74), tienen un gasto energético menor cuando realizan la misma rutina de porrismo (Soriano y Belloch, 2016).

En cuanto a la segunda pregunta de investigación, el gasto energético se midió con acelerómetros y pulsómetros, los cuales realizan el cálculo por medio de diferentes algoritmos, en conjunto con la información de cada sujeto, como edad, peso, talla, sexo, entre otros.

Es importante recalcar que las mediciones de FC y acelerometría son diferentes formas de medir la actividad física, mientras que las mediciones de FC dependen de la aptitud física del participante. El acelerómetro mide el movimiento absoluto independientemente de la aptitud del participante (Dyrstad y Hausken, 2014). Los datos recolectados en el presente estudio evidenciaron una correlación significativa, moderada y directa $\left(\mathrm{r}=0.45, \mathrm{p} \leq 0.001^{*}\right)$ entre el gasto energético registrado por los acelerómetros y el gasto energético registrado por los pulsómetros. Sin embargo, los registros de ambos instrumentos son significativamente diferentes $\left(\mathrm{F}=46.9, \mathrm{p}=\mathrm{p} \leq 0.001^{*}\right)$, es decir, el gasto energético mostrado por los pulsómetros fue significativamente mayor que el registro de los acelerómetros. Debe quedar claro que, ante la limitada disponibilidad de los dispositivos en el centro de investigación, no a todos los sujetos se les colocó pulsómetro $(\mathrm{N}=65)$.

Esta diferencia podría explicarse principalmente porque se ha visto que en actividades de intensidad vigorosa (como la que se registró en el presente estudio) se tiende a sobreestimar el gasto calórico si solo se toma como referencia la FC, esto debido a que el sistema cardiovascular necesita unos minutos para regularse después de un cambio repentino de intensidad, por lo que en los momentos de reposo el registro sigue elevado hasta que la FC vuelva a regularse (Dyrstad y Hausken, 2014). Esto es justamente lo que sucede en los entrenamientos de porrismo, en donde se presenta una máxima exigencia física durante la ejecución de las rutinas pero, una vez terminadas, los atletas vuelven al estado de reposo para volver a ejecutarla en 1 os siguientes minutos. La justificación de la utilización del método con pulsómetros es que la medición del gasto energético sin tomar en cuenta la FC (como en el método con acelerómetros), por el contrario, tiende a subestimar la carga de trabajo de entrenamiento, pero realiza una medición más precisa en los momentos de reposo, ya que reaccionan instantáneamente al inicio-parada (McArdle et al., 2010), por lo que para el presente estudio fue muy importante emplear ambos métodos de medición.

Ahora bien, de acuerdo con la información recolectada con los acelerómetros, se evidenció una interacción s ignificativa $\left(\mathrm{p}=0.008^{*}\right)$ entre ambas variables independientes (figura 2$)$, la cual muestra que los hombres de categoría de gimnasia "alta" tuvieron un gasto calórico $(\overline{\mathrm{X}}=300.9 \mathrm{kcal})$ significativamente superior al de 1 os hombres de categoría "media” ( $\bar{X}=178.5 \mathrm{kcal}$ ) y "baja” ( $\overline{\mathrm{X}}=180.4 \mathrm{kcal})$, y aún más que las mujeres de t odas la categorías (“alta” $\bar{X}=151.3 \mathrm{kcal}$; “media” $\overline{\mathrm{X}}=153.5 \mathrm{kcal}$; “baja” $\overline{\mathrm{X}}=146.8 \mathrm{kcal}$ ). Por otro lado, 1 a información brindada por los pulsómetros mostró una diferencia significativa $\left(p=0.048^{*}\right)$ entre sexos ( figura 3), es decir, los hombres tuvieron un mayor gasto calórico que las mujeres.

Debido a la limitada producción de estudios con porristas, es difícil realizar una comparación de resultados con otra muestra compuesta por el mismo tipo de deportistas. Sin embargo, vale la pena compararlo con un estudio como el de Cugusi et al. (2017), quienes midieron un entrenamiento en trampolín categorizado como actividad física media-alta (5.2 METs) y, con una duración aproximada de 50 min, ellos evidenciaron 
un gasto energético promedio de $317 \mathrm{kcal}$; efectuaron dichas mediciones con acelerómetros y pulsómetros. Si bien las muestras no se asemejan, podría decirse que el gasto energético de las dos actividades físicas fue relativamente cercano, sin olvidar que el gasto energético del entrenamiento mostrado por porristas fue más elevado. En este sentido, esta comparación es relevante porque el tiempo de registro fue muy similar al del presente estudio; además, el entrenamiento en trampolín también se utiliza en porrismo, para mejorar sus habilidades gimnásticas y, también es un entrenamiento que exige el trabajo de la mayoría de los grupos musculares, lo cual es similar a lo que sucede en los entrenamientos de porrismo.

A partir de los resultados del presente estudio, se podría presumir que los hombres que poseen gimnasia de mejor nivel presentan mayores gastos calóricos en los entrenamientos de porrismo y, quizás, también en el momento de competir. Desde el punto de vista fisiológico, la diferencia significativa entre sexos podría explicarse principalmente por la diferencia del porcentaje de masa muscular relativo al peso corporal, pues se sabe que por un factor hormonal (testosterona) los hombres poseen mayor masa muscular que las mujeres y, que a mayor masa muscular mayor gasto calórico (McArdle et al., 2010). Desde el punto de vista técnico-deportivo, en los equipos en general, la cantidad de hombres que poseen un alto nivel de gimnasia es mayor que las mujeres con dicho nivel de gimnasia, como sucedió en con la muestra reclutada en el presente estudio ( $\mathrm{N}$ de hombres con gimnasia "alta" = 29; $\mathrm{N}$ de mujeres con gimnasia "alta" $=8$ ), por lo que esto también podría explicar la diferencia entre sexos.

En cuanto a la intensidad, no se evidenciaron diferencias significativas ( $p>0.05$ ) en las variables FCP y FCmax entre categoría de gimnasia y sexo. Los registros de FCP mostraron un promedio de $132 \mathrm{lpm}$, lo que se traduce en que los atletas se mantuvieron alrededor del 70\% de la FCmax durante todo el entrenamiento de rutinas completas. Además, se registró un promedio de FCmax de 197 lpm, lo que representa el 102\% de la FCmax en promedio; es importante mencionar que un sujeto llegó a 240 lpm (124\% de la FCmax) y 7 sujetos registraron FCmax mayor a $230 \mathrm{lmp}$ (122\% de la FCmax en promedio).

En este sentido, la FCP mostrada en el presente estudio se mantuvo por debajo de la FCP de una muestra de porristas que realizó un entrenamiento en trampolín, pues se reportó una intensidad del 70\%-85\% de la frecuencia cardiaca máxima. Dicho entrenamiento tuvo una duración aproximada de 45 min (Ulloa y Hernández, 2020), que es algo parecido a la duración del entrenamiento de rutinas completas medido en el presente estudio; esta comparación viene al caso por cuanto, como se mencionó, este tipo de entrenamiento también es parte de la formación en porrismo. El hecho de que la FCP fuera más baja en comparación con el estudio de Ulloa y Hernández (2020) se puede explicar porque en el entrenamiento de trampolín los tiempos de recuperación fueron relativamente cortos y bien sistematizados, siguiendo una rutina específica, mientras que en el entrenamiento de rutinas completas los tiempos de recuperación fueron más extensos y variables. Eso afectaría el dato final de FCP; pero, sin duda, la intensidad máxima expresada por la FCmax de las rutinas completas fue mayor que el ET.

También podría compararse con ejercicios globales o ejercicios que simulan circunstancias de juego de baloncesto. En este sentido, Delextrat y Kraiem (2013) evidenciaron que estas pruebas deportivas mantienen la FC entre el $87 \%$ y $92 \%$ de la frecuencia cardiaca máxima; incluso Ainsworth et al. (2011) reportan que este deporte eleva el gasto metabólico a 7 METs, es decir, se clasifica como actividad física vigorosa. Conviene tomar en cuenta que dichos ejercicios no duraron una hora como el entrenamiento de rutinas completas, por lo que lo más idóneo sería compararlo con la FCmax de los sujetos del presente estudio, en el que la ejecución de rutinas completas fue más intenso (entre 70-124\% FCmax) que los ejercicios globales del baloncesto.

Las FC tan elevadas (principalmente las FCmax) evidencian que la ejecución de una rutina o, como en este caso, varias rutinas de porrismo en un mismo entrenamiento exige un esfuerzo físico máximo, pues a pesar de que las rutinas solo duran dos minutos y medio, desde el primer segundo la ejecución involucra elementos y técnicas que requieren de fuerza explosiva de muchos grupos musculares en conjunto y, además, deben ejecutarlos con la mejor precisión posible. 
Tomando en cuenta lo anterior, se podría pensar que medir únicamente el tiempo efectivo en que las personas atletas ejecutan estrictamente las rutinas conllevaría a que tanto las FCP como el gasto energético en términos de METs tuvieran valores más altos; una evidencia que apoyaría dicha hipótesis son los dos sujetos del presente estudio que registraron 8.1 y 8.7 METs: esto mostraría más específicamente el nivel de exigencia física que experimentan los porristas al ejecutar sus rutinas, y vale la pena mencionarlo a pesar de que no fue el objetivo del estudio.

\section{Conclusiones}

1. El dato de equivalentes metabólicos reportado en el compendio de gasto energético de Ainsworth et al. (2011) para el porrismo (6 METs) es equivalente al gasto energético registrado en los entrenamientos de porrismo $(\overline{\mathrm{X}}=5.8 \mathrm{METs}, \mathrm{N}=103)$.

2. Existe una interacción significativa entre categoría de rendimiento (categoría de gimnasia) y sexo cuando se analiza el gasto energético en un entrenamiento de porrismo, siendo los hombres con categoría "alta" de gimnasia los que tienen un gasto energético significativamente mayor $\left(\mathrm{p}<0.05^{*}\right)$ comparado con los hombres de categoría de gimnasia "media" y "baja” y, con el de las mujeres de las tres categorías de gimnasia.

3. No hay diferencias significativas de la intensidad (FCP y FCmax) en el entrenamiento de porrismo entre categoría de rendimiento (categoría de gimnasia) y sexo.

\section{LIMITACIONES}

Una de las principales limitaciones del presente estudio fue que no se pudo realizar una distribución más homogénea de pulsómetros entre las diferentes categorías de rendimiento de gimnasia (bajo, medio, alto), debido a que la mayoría de los sujetos brindaron la información de su nivel de gimnasia en el mismo momento de la recolección de datos, por lo que se optó por una asignación aleatoria para determinar los que los usarían.

Otra limitante fue que la acelerometría y la frecuencia cardiaca no se emplearon en conjunto para realizar los cálculos de gasto energético, sino que, por una limitación tecnológica, se utilizaron por separado.

\section{RECOMENDACIONES}

Sería aconsejable realizar este tipo de mediciones en los festivales y/o eventos en los que los equipos de porrismo simulan una competencia, como es muy común en Costa Rica. Además, dichas mediciones deberían complementarse con mediciones en competiciones reales; con esto podrían evidenciarse posibles diferencias en variables tanto fisiológicas como psicológicas.

\section{Aplicaciones práCticas}

De acuerdo con los resultados del presente estudio y tomando en cuenta que las rutinas tienen una duración estándar de 2 min y $30 \mathrm{~s}$, la preparación física de este deporte debe basarse en el entrenamiento del sistema energético anaeróbico, específicamente la resistencia anaeróbica ( $\mathrm{ATP}+\mathrm{PCr}+$ ácido láctico), ya que es el principal sistema energético implicado en dicha práctica.

En este sentido, debido a que en la actualidad el material bibliográfico-práctico para entrenamiento de porrismo es escaso, se recomienda seguir el principio de especificidad del entrenamiento, la cual explica que las adaptaciones en las funciones metabólicas y fisiológicas dependen de la intensidad, duración, frecuencia y modo de sobrecarga impuesta. En otras palabras, el entrenamiento y la preparación física en porrismo 
deberían enfocarse en la ejecución sus fundamentos técnicos emulando los niveles de exigencia física de las rutinas, tal como quedó evidenciada (en términos de intensidad y gasto energético) en el presente estudio.

Además, es fundamental tener claros los principios del entrenamiento anaeróbico. En el caso específico de los entrenamientos de rutinas completas, cada ejecución demanda un esfuerzo fisiológico y psicológico casi al máximo, por lo que es indispensable respetar los periodos de recuperación, para aminorar los efectos negativos de la fatiga central (sistema nervioso central) y periférica (acumulación de metabolitos) cuanto sea posible, para optimizar el rendimiento (Haff y Triplett, 2015; McArdle et al., 2015). Cabe recordar que el porrismo es un deporte estético, es decir, busca la perfección de sus ejecuciones, por lo que estaría contraindicado ejecutar las rutinas de competencia sin una aptitud física y mental óptima.

\section{Agradecimientos}

Al Centro de Investigación en Ciencias del Movimiento Humano (CIMOHU) y a la Escuela de Educación Física y Deportes (EDUFI) de la Universidad de Costa Rica, por el apoyo brindado para la ejecución de este proyecto.

A atletas, entrenadores, entrenadoras y personal administrativo de los programas de porrismo Pura Vida Athletics, Jungle Cats, Cheer Company Pirates y la Federación Cheer and Dance Costa Rica (FECAD) Federación Cheer and Dance Costa Rica (FECAD), sitio web: https://www.fecadcostarica.org/, por facilitar amablemente sus instalaciones y entrenamientos para la recolección de datos.

No se declara conflictos de interés.

\section{ReFERENCIAS}

Ainsworth, B. E., Haskell, W. L., Herrmann, S. D., Meckes, N., Bassett, D. R., Tudor-Locke, C., Greer, J. L., Vezina, J., Whitt-Glover, M. C. \& Leon, A. S. (2011). 2011 Compendium of Physical Activities: A second update of codes and MET values. Medicine and Science in Sports and Exercise, 43(8), 1575-1581. https://doi.org/10.124

9/MSS.0b013e31821ece12

Cugusi, L., Manca, A., Romita, G., Bergamin, M., Di Blasio, A., \& Mercuro, G. (2017). Exercise intensity and energy expenditure during a mini-trampoline rebounding exercise session in overweight women. Science \& Sports, 32(1), e23-e28. https://doi.org/10.1016/j.scispo.2016.06.006

Delextrat, A., \& Kraiem, S. (2013). Heart-rate responses by playing position during ball drills in basketball. International Journal of Sports Physiology and Performance, 8(4), 410-418. https://doi.org/10.1123/ijspp.8.4.4 10

Dyrstad, S. M., \& Hausken, K. (2014). Comparing Accelerometer and Heart Rate Monitor in Interval Running, Interval Spinning and Zumba. International Journal of Applied Sports Sciences, 26(2), 89-98.

Haff, G. G. \& Triplett, N. Travis. (2015). Essentials of Strength Training and Conditioning (4. ed.). Human Kinetics. International Cheer Union [ICU]. (2020). History of Cheerleading. http://cheerunion.org/home/

Latzel, R., Hoos, O., Stier, S., Kaufmann, S., Fresz, V., Reim, D. \& Beneke, R. (2018). Energetic profile of the basketball exercise simulation test in junior elite players. International Journal of Sports Physiology and Performance, 13(6), 810-815. https://doi.org/10.1123/ijspp.2017-0174

Leppänen, M. H., Migueles, J. H., Cadenas-Sánchez, C., Henriksson, P., Mora-González, J., Henriksson, H., Labayen, I., Löf, M., Esteban-Cornejo, I., \& Ortega, F. B. (2019). Hip and wrist accelerometers showed consistent associations with fitness and fatness in children aged 8-12 years. Acta Paediatrica, 109(5), 995-1003. https://d oi.org/10.1111/apa.15043

Lyden, K., Keadle, S. K., Staudenmayer, J., Freedson, P., \& Alhassan, S. (2013). Energy Cost of Common Activities in Children and Adolescents. Journal of Physical Activity \& Health, 10(1), 62-69. https://doi.org/10.1123/jp ah.10.1.62 
MHSALUD, ISSN: 1659-097X, 18(2), JuLio-Diciembre, 2021, PP 1-14

Ulloa SÁnchez, Hernández Elizondo

McArdle, W. D., Katch, F. I., \& Katch, V. L. (2010). Exercise Physiology: Nutrition, Energy, and Human Performance. Lippincott Williams \& Wilkins.

McArdle, W. D., Katch, F. I., \& Katch, V. L. (2015). Fisiología del ejercicio: Nutrición, rendimiento y salud (8. ed.). Wolters Kluwer Health.

Soriano, P. P., \& Belloch, S. L. (2016). Biomecánica básica: Aplicada a la actividad física y el deporte (Color). Paidotribo.

Tanaka, H., Monahan, K. D., \& Seals, D. R. (2001). Age-predicted maximal heart rate revisited.Journal of the American College of Cardiology, 37(1), 153-156. https://doi.org/10.1016/S0735-1097(00)01054-8

Ulloa, P., \& Hernández, J. (2020). El efecto agudo del entrenamiento en trampolín sobre la estabilidad, la altura de salto y la velocidad de carrera en 20 metros de porristas. Pensar en Movimiento: Revista de Ciencias del Ejercicio y la Salud, 18(1), e38560-e38560. https://doi.org/10.15517/pensarmov.v18i1.38560

US Department of Health and Human Services (2008). Physical activity guidelines for Americans: Be active, healthy, and happy! http://www.health.gov/paguidelines/guidelines/default.aspx

Zakeri, I., Puyau, M. R., Adolph, A. L., Vohra, F. A., \& Butte, N. F. (2006). Normalization of energy expenditure data for differences in body mass or composition in children and adolescents. The Journal of Nutrition, 136(5), 1371-1376. https://doi.org/10.1093/jn/136.5.1371

Zunzer, S. C., von Duvillard, S. P., Tschakert, G., Mangus, B., \& Hofmann, P. (2013). Energy expenditure and sex differences of golf playing. Journal of Sports Sciences, 31(10), 1045-1053. https://psycnet.apa.org/doi/10.108 $0 / 02640414.2013 .764465$ 